\title{
Structural Vibration Monitoring Using Cumulative Spectral Analysis
}

\author{
Satoru Goto, ${ }^{1}$ Yoshinori Takahashi, ${ }^{2}$ and Mikio Tohyama ${ }^{1}$ \\ ${ }^{1}$ Wave Science Study, 2-7-8 Fujigaya, Kugenuma, Fujisawa-shi, Kanagawa 251-0031, Japan \\ ${ }^{2}$ Tokyo Metropolitan College of Industrial Technology, 8-17-1 Minami Senju, Arakawa-ku, Tokyo 116-0003, Japan \\ Correspondence should be addressed to Satoru Goto; ahjinsei@yahoo.co.jp
}

Received 15 November 2012; Accepted 28 January 2013

Academic Editor: K. M. Liew

Copyright (c) 2013 Satoru Goto et al. This is an open access article distributed under the Creative Commons Attribution License, which permits unrestricted use, distribution, and reproduction in any medium, provided the original work is properly cited.

\begin{abstract}
This paper describes a resonance decay estimation for structural health monitoring in the presence of nonstationary vibrations. In structural health monitoring, the structure's frequency response and resonant decay characteristics are very important for understanding how the structure changes. Cumulative spectral analysis (CSA) estimates the frequency decay by using the impulse response. However, measuring the impulse response of buildings is impractical due to the need to shake the building itself. In a previous study, we reported on system damping monitoring using cumulative harmonic analysis (CHA), which is based on CSA. The current study describes scale model experiments on estimating the hidden resonance decay under non-stationary noise conditions by using CSA for structural condition monitoring.
\end{abstract}

\section{Introduction}

Structural health condition diagnostics are needed for ensuring the safety of buildings struck by earthquakes and old buildings. Spectral analysis focuses on the change in the resonant frequency caused by structural degradation, and it yields useful information for health monitoring. However, for structural health monitoring, spectral analysis requires a test signal. Although using a test signal is an easy way to measure structural spectral characteristics, it is impractical to vibrate a structure during every measurement. If the spectral characteristics could be measured using structural vibrations due to wind, ground motion, and so forth, the health of the structure would be able to be monitored all the time. However, this sort of measurement would require a lot of spectral information about external noise characteristics besides the structural spectral characteristics. For this reason, spectral characteristics analysis for structural health monitoring generally requires the external source characteristics to be known at all times $[1,2]$.

Hirata proposed the short-interval period (SIP) distribution to analyze the dominant spectral components of a structure subject to an unknown vibration or even nonstationary noise [3]. The SIP distribution represents the shape of the frequency response in poor measuring conditions. Recently, Hirata et al. showed that the SIP distribution can be used to get the gradual time variation of the frequency response of a structure subjected to nonstationary vibrations [4]. On the other hand, the SIP distribution requires a sufficiently long signal because the computational process of the SIP distribution uses information on the dominant frequency components taken from a number of short intervals.

Takahashi et al. proposed a health monitoring method that focuses on damping using cumulative harmonic analysis (CHA) to reveal the spectral peaks of hidden structural resonances in short signals [5]. In a recent report, the authors estimated the temporal damping changes in the presence of unknown nonstationary vibrations by using the interpolated power spectrum of CHA [6]. Damping is an important factor in structural diagnostics, and it is related to the resonant decay of transient information in the time domain. For instance, if a pillar has a crack, any resonance decay will be short lived. This means that a way of estimating transient information such as resonant decay would be of value to structural diagnostics. CHA is an extension of cumulative spectral analysis (CSA) proposed by Berman and 
Condition 1
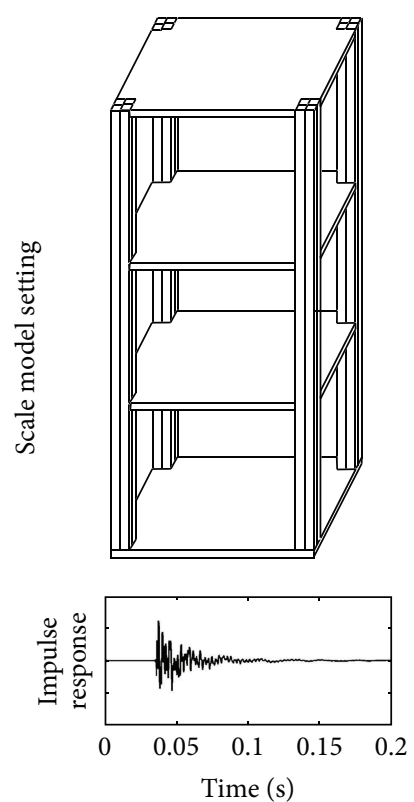

Condition 2

Linear interpolation to simulate structural degradation
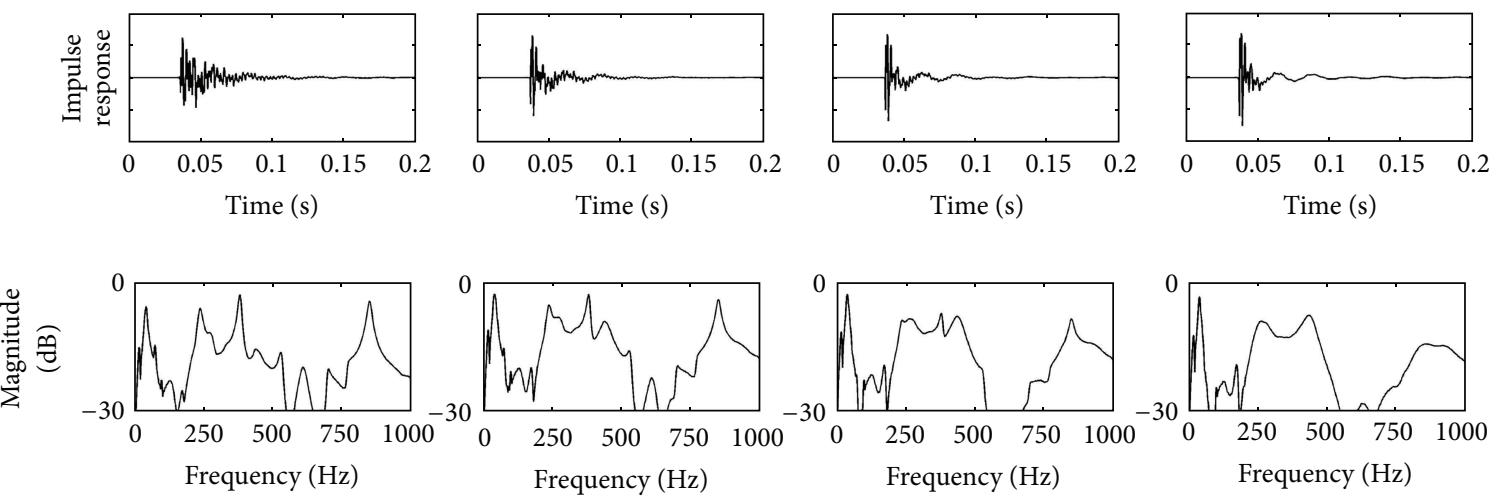

FIGURE 1: Scale models and corresponding measured and interpolated impulse responses and magnitude spectra.

Fincham [7], and CSA determines the transient characteristics of loudspeaker systems. In terms of the relationship between transient information and CHA, Takahashi et al. predicted the howling frequency by using a histogram of $\mathrm{CHA}$ power spectrum peaks of the transient decaying portion [8]. Thus, in acoustic systems, spectral analysis with a stepwise time window can accurately detect resonance peaks under the unknown signals and possibly predict the hidden frequency change from the transient decaying portions of the histogram of the CHA peaks in a simple feedback system. Consequently, when using a stepwise time window spectral analysis, we assume that acoustical techniques could be applied to structural analysis in order to reveal the resonance decay characteristics for structural diagnostics under nonstationary noise.

In this paper, we discuss a hidden resonance decay estimation for structural health monitoring that does not need the frequency characteristics of the external noise. We also describe experiments on scale models subjected to simulated nonstationary noise that assessed the effectiveness of resonance decay estimation focused on the transient decay portion.

Section 2 describes the measurement of the scale model's impulse response. Section 3 explains the CSA analysis and impulse response analysis. Section 4 describes the experiments in ideal cases of resonance decay analysis on the decay portions of the spectra. Section 5 discusses the results of the simulation experiment. Section 6 discusses the possibility of quantitative structural diagnostics using experimental CSA results.

\section{Scale Model and Impulse Responses for a Simulation Experiment}

We made two scale models and measured their impulse responses. Each scale model was a wooden framework $(18 \mathrm{~cm}$ $(W) * 21 \mathrm{~cm}(D) * 38 \mathrm{~cm}(H))$ of a three-story building. The scale model had four pillars, and each pillar comprised $1 \mathrm{~cm}$ square lumber. Impulse responses were recorded at an Fs of $6 \mathrm{kHz}$ using a piezoelectric accelerometer (PV-90B, RION) with an impulsive hammer. Figure 1 shows the scalemodel experimental conditions and the linear interpolated impulse response waveforms and magnitude responses. As the upper illustration of Figure 1 shows, we prepared two scale models for condition 1 and condition 4 . Conditions 2 and 3 simulated structurally degraded conditions by linear interpolation between the impulse response waveforms of conditions 1 and 4. The lower panels show the impulse response waveforms and magnitude spectra. From these panels, we can see that the magnitude spectra characteristics gradually change from condition 1 to condition 4 . 

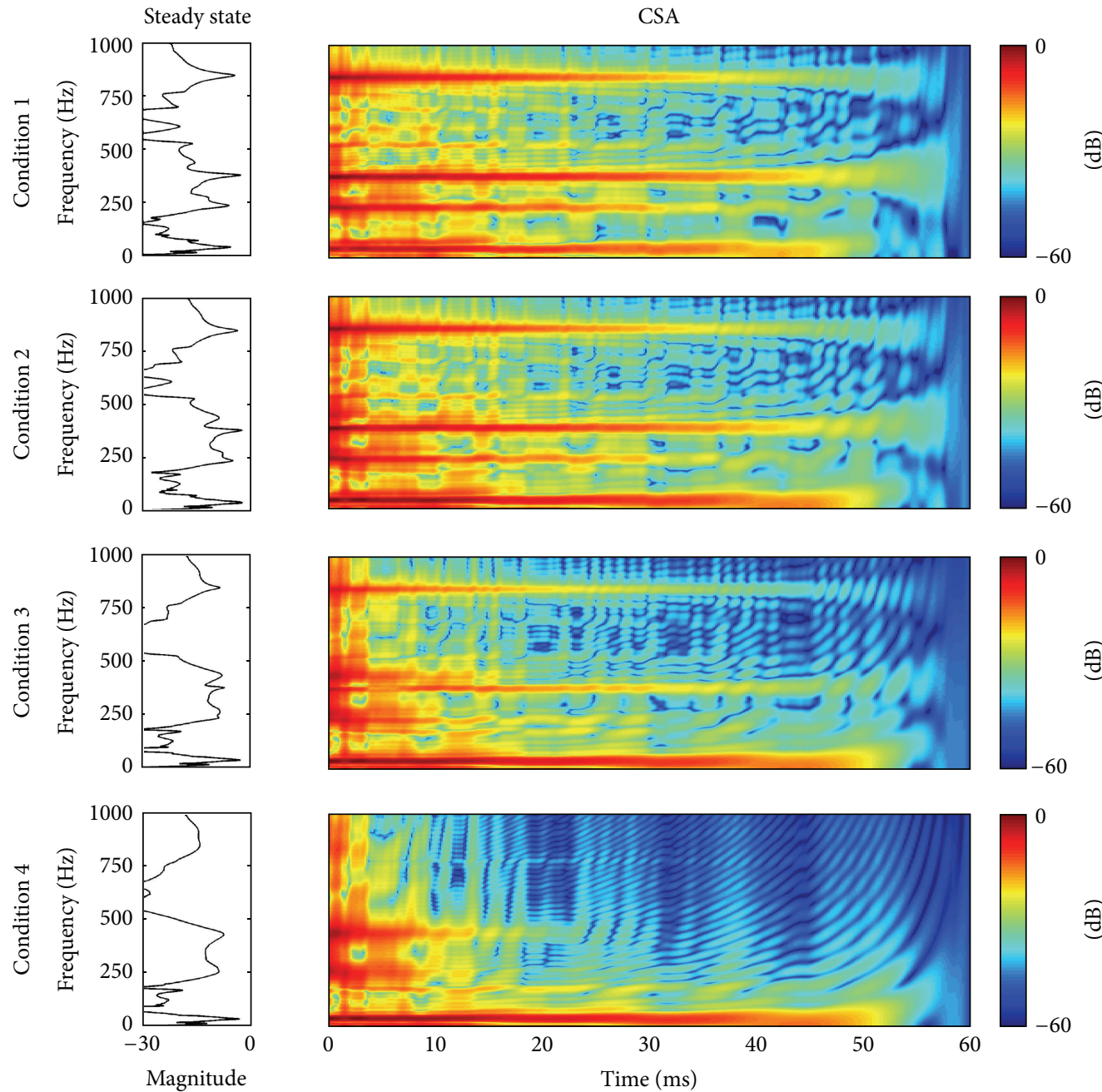

$(\mathrm{dB})$

Figure 2: CSA of impulse responses.

\section{Spectral Analysis with Stepwise Time Window}

We used stepwise time window spectral analysis to make the resonance decay estimation. The cumulative spectral analysis (CSA) of a framed input signal $x(n)$ is formulated as

$$
\operatorname{CSA}(n, k)=\sum_{m=n}^{N-1} u(m) x(m) e^{j(2 \pi k / N) m} \quad 0 \leq n \leq N-1 .
$$

Here, $u(n)$ is a unit-step function corresponding to input signal conditions such as

$$
u(n)= \begin{cases}1, & 0 \leq n \\ 0, & n<0\end{cases}
$$

We set $N$ to include zero padding of 10 times the frequency interpolation of Fs.

Figure 2 shows the CSAs of the impulse responses corresponding to the different structural conditions. We calculated the CSAs by using a $60 \mathrm{~ms}$ frame to extract the impulse response decay portion. The left panel shows the magnitude spectrum of the impulse responses in the steady state, whereas the right panel illustrates the results of CSA. This figure shows that the spectral peaks in the magnitude spectra are the same as those in the CSA results. In addition, if there is a peak on the time axis, the changes in the decay of the resonant frequency can be read in the CSAs across conditions. Moreover, the fundamental frequency of $45 \mathrm{~Hz}$ does not decay in any of the plots. This is because the structure profile of the scale model did not change in these experiments. Therefore, the fundamental frequency did not decrease much compared with the higher resonances. The effect of CSA can be seen by comparing the peaks above $250 \mathrm{~Hz}$. As for the peak at $850 \mathrm{~Hz}$, it lasts for $40 \mathrm{~ms}$ under condition 1 . Moreover, the time period of the peak gradually decreases to under $5 \mathrm{~ms}$ in going from condition 1 to condition 4. From these results, we supposed that CSA can estimate resonance peaks and their decays even when the decay portion is shorter than the impulse response. 


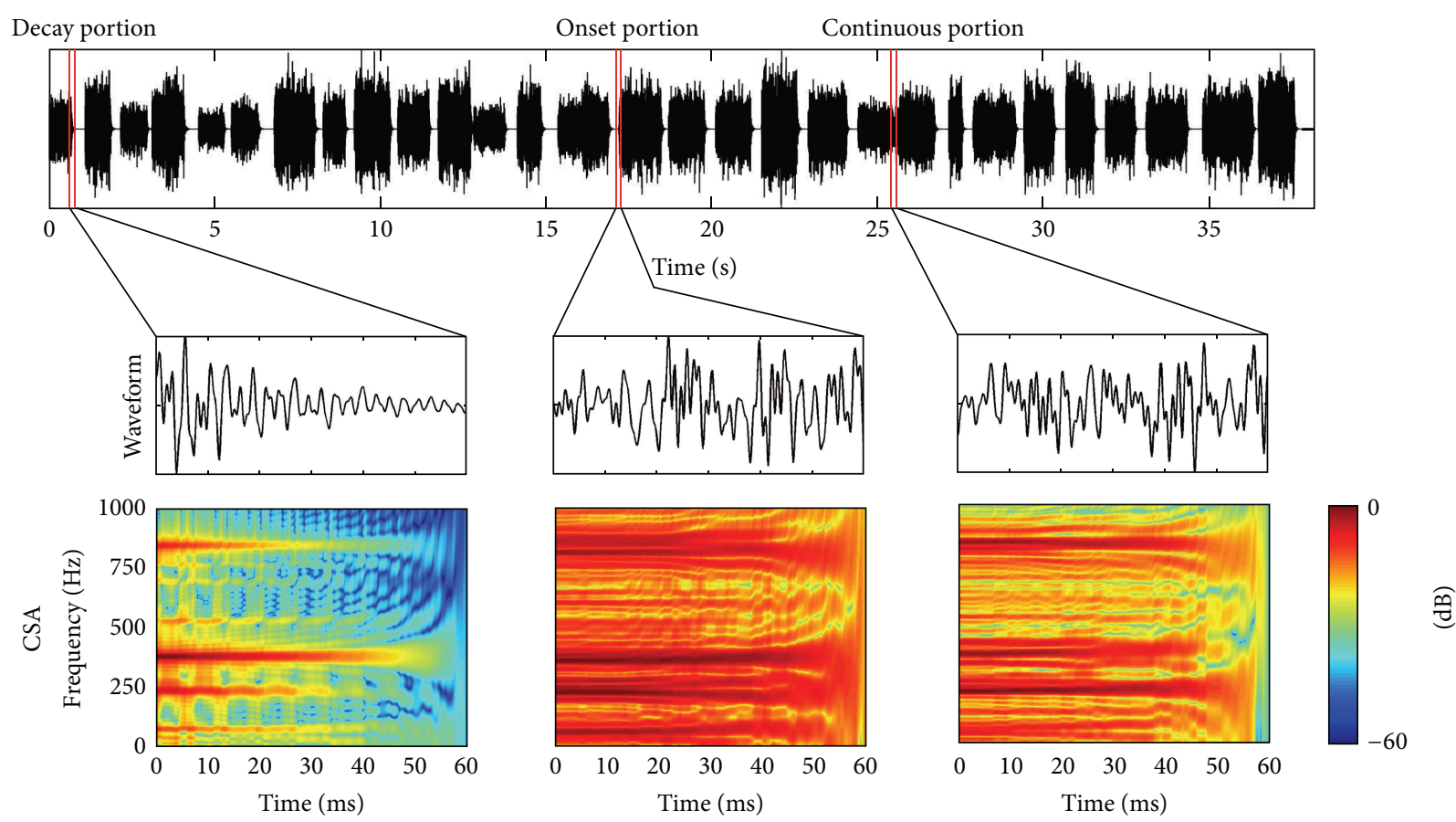

FIgURE 3: Random burst noise and CSA of different three portions.

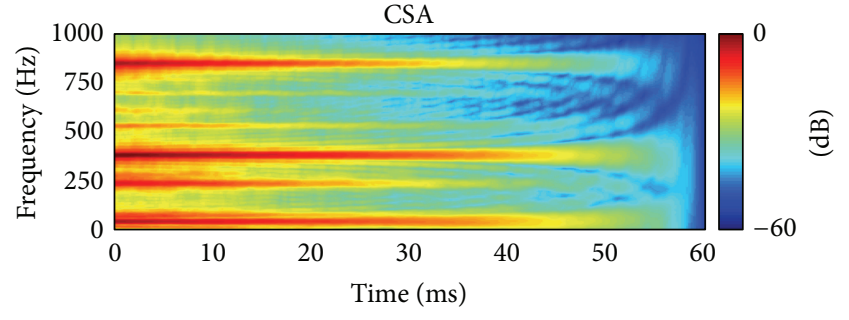

Figure 4: Mean of CSAs on all decay portions in the presence of random noise bursts.

\section{Random Burst Noise Analysis for the Ideal Case of Resonance Decay}

When a vibration stops, a transient decay arises through the system's transfer function. This transient decay includes the effect of the system's impulse response. To simulate this transient decay under ideal circumstances, we prepared random burst white noise having random durations, intervals, and magnitudes.

Figure 3 shows a random burst noise waveform convolved with the impulse response measured under condition 1 and the CSAs of different extracted portions. The upper panel illustrates the random burst noise and highlights of the extracted waveform for different portions. The decay portion on the left side is just after the random noise stopped. The onset portion in the middle is the onset of the convolved random noise. The continuous portion on the right side is the mixture of the decay of the signal and the beginning of the next random noise. The corresponding waveforms are in the second top panels. CSA at the bottom reveals different resonance decays between the decay portion and the other portions. The CSA of the decay portion has similar characteristics to those of the CSA of condition 1's impulse response in Figure 2. This means that the frequency characteristics including spectral decay can be estimated by using CSA on the decaying signal portion. On the other hand, the CSAs of the onset and continuous portions do not have spectral decays. This is because in the onset and continuous portions, the input signal was continuous and an increase in the resonance continued. This means that if the signal is found in the decay potion, CSA will reveal a hidden resonance decay.

Figure 4 shows the mean of the CSAs of the decaying portions of the whole simulated signal. In this figure, we can see that the magnitude spectrum of the impulse response and mean of CSA have the same spectral peaks, even the small peaks between 500 and $750 \mathrm{~Hz}$. Furthermore, comparing this with the decay portion in Figure 3, the decay of the fundamental frequency is close to that of the CSA results of the impulse response in Figure 2. Accordingly, we can see that the mean of CSA is useful for estimating the resonant decay of the structure even in a short-frame signal such as $60 \mathrm{~ms}$. Therefore, if constant monitoring is possible for structural health monitoring, the mean of the decay portions' CSAs could be used to show the structural resonance frequencies and their decays.

\section{Structural Degradation Estimation Using Simulated Signals}

In most settings, the signal length of the impulse response for sound sources such as musical instruments is short. 


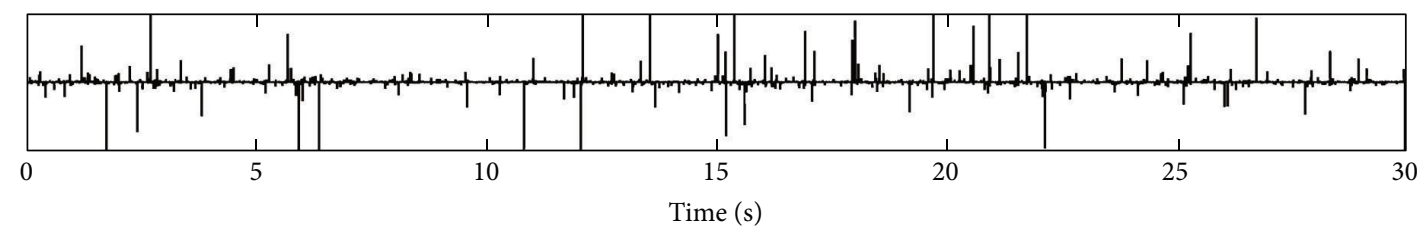

FIGURE 5: Simulated nonstationary noise waveform.

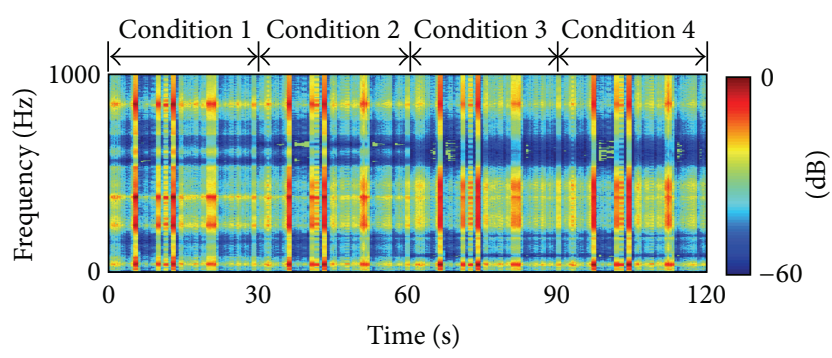

FIGURE 6: Spectrogram of simulated observed signals.

In contrast, structural impulse responses or reverberations of structures or halls are longer because the length of the impulse response depends on the system size and resonance, and the sound source is usually much smaller than the whole system. Therefore, if the observed signals contain a decay portion, it has a lot of information on the structural characteristics related to the impulse response of the system. In this experiment, we prepared the nonstationary noise and convolved it with the simulated structurally degrading impulse responses. The simulated signals were analyzed and the mean of the decay portion CSAs were calculated in order to show the resonance frequencies and their decays.

We prepared simulated nonstationary noise which had a Cauchy distribution. The simulated nonstationary noise $C(n)$ is defined as

$$
C(n)=\frac{G_{A}(n)}{G_{B}(n)},
$$

where $G_{A}(n)$ and $G_{B}(n)$ each mean Gaussian noise.

Figure 5 shows the waveform of the simulated nonstationary noise, and we can see sudden vibrations in it. After the impulse responses were convolved with the nonstationary noise as simulated observed signals, we used the sudden vibrations to extract the transient decay portions. To confirm the spectral changes in a simulated observed signal, we joined 30 seconds of the simulated observed signals for each condition in sequence. Figure 6 shows a spectrogram using a 1 -second framed DFT with a 0.5 second overlap of the joined signals. This figure shows the difficulty of revealing spectral changes under nonstationary noise conditions. Moreover, because of the large changes in energy due to the precipitous vibration, it is difficult to discriminate between conditions in the spectrogram. Regarding the resonant decay, such a spectrogram cannot be seen due to the poor time resolution. Putting these facts together, it is clear that a finer analysis is required for estimating the resonance frequency and decay in
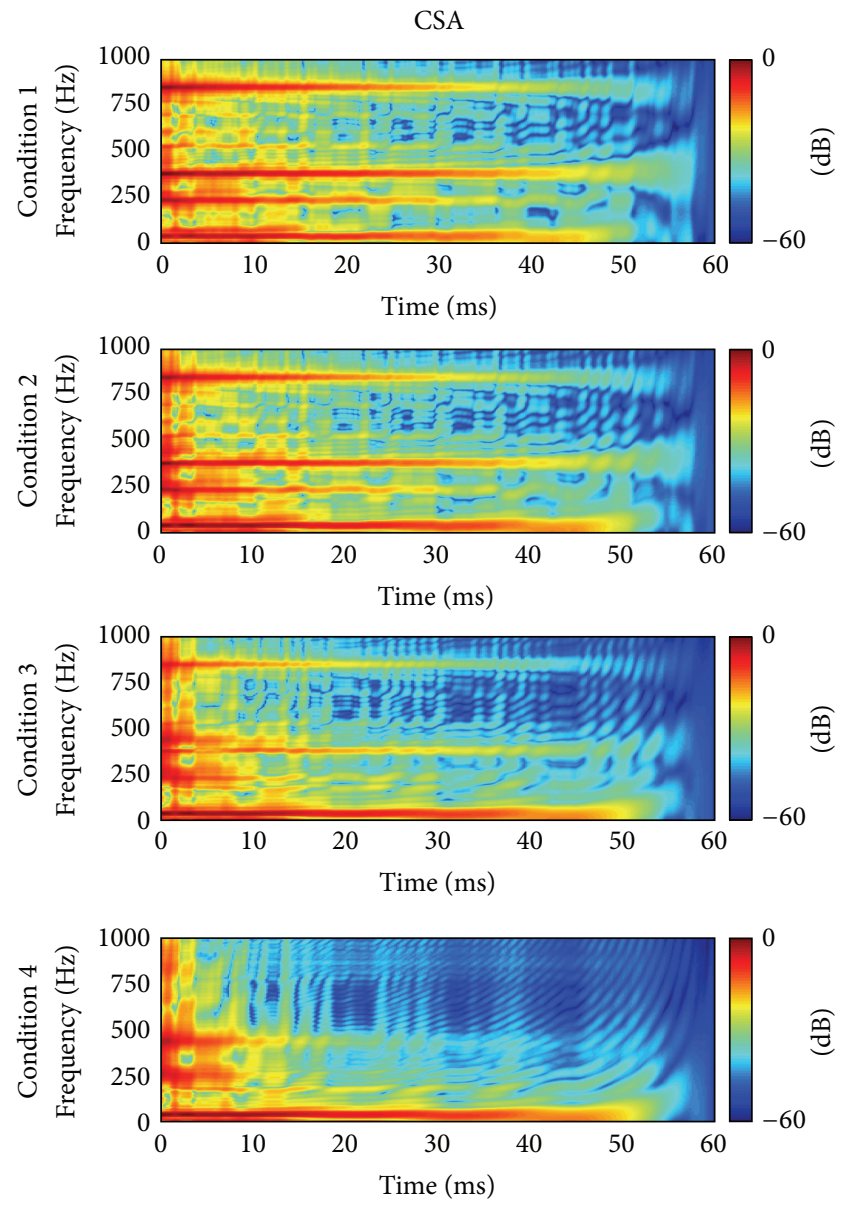

FIGURE 7: Mean of CSAs of decay portions in simulated observed signals.

the presence of nonstationary noise and without knowing the external source characteristics. Accordingly, we conducted resonance frequency and decay estimations using the CSAs of the decay portions.

In this experiment, we extracted 16 decay portions from the simulated observed signals for sudden vibrations in the order of the largest to the sixteenth largest. Figure 7 shows the mean CSAs of the decaying portions of the simulated observed signals for each condition. The resonant frequencies and their decays are very similar to the impulse response CSAs in Figure 2. Moreover, we can see that the durations of the peaks above the $250 \mathrm{~Hz}$ gradually decrease from condition 1 to condition 4 . Also, the fundamental frequency and the decay duration were the same for all conditions. 


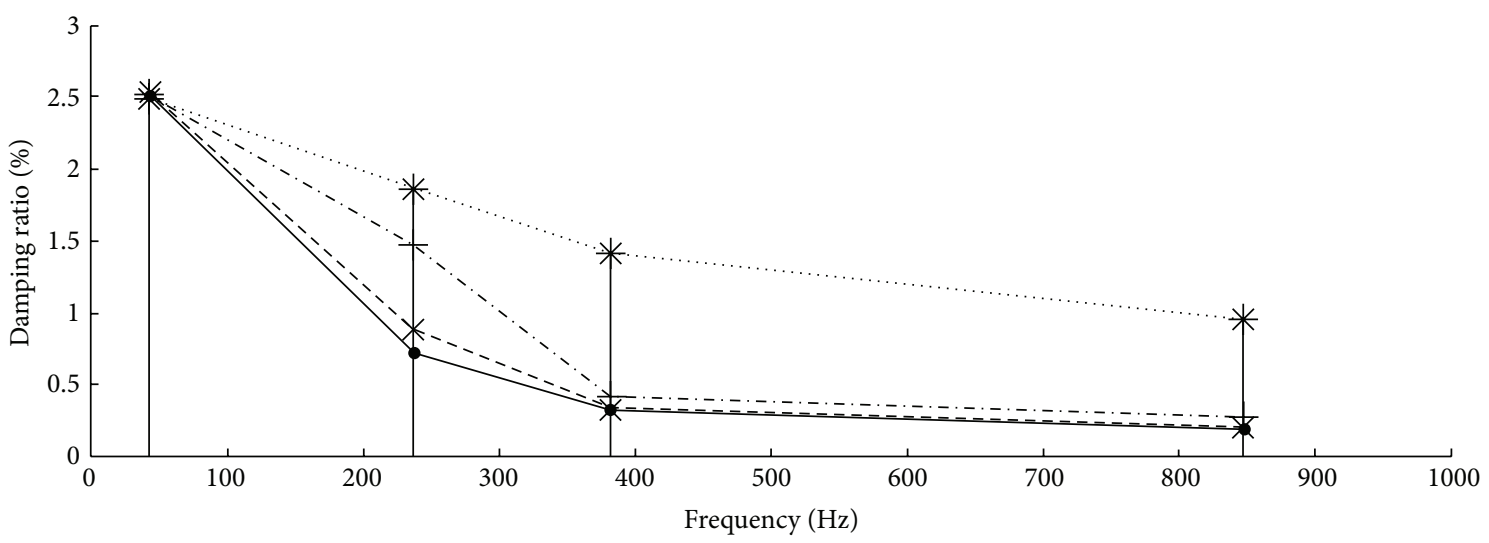

$\rightarrow$ Condition 1

$-\rtimes-$ Condition 2

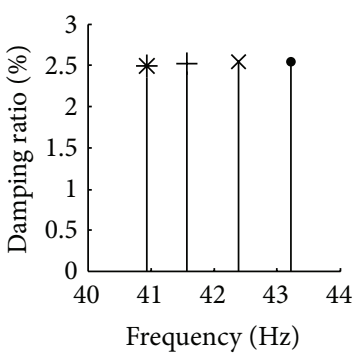

- Condition 1

$\times$ Condition 2
+- Condition 3

*. Condition 4
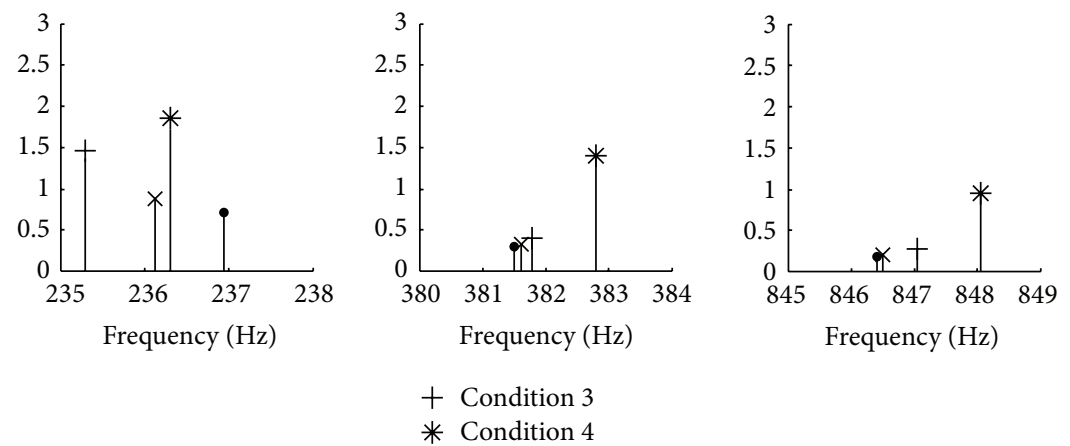

FIGURE 8: Damping ratio calculated from simulated observed signals' CSAs.

This means that the resonance changes for structural health monitoring are reliable even in the presence of nonstationary noise. Consequently, the CSAs of the decay portions can reveal structural changes in a constantly monitored signal without us having to know the noise spectral characteristics.

\section{Discussion}

The results of the previous experiment revealed the hidden resonance frequencies and their decays under unknown nonstationary signal conditions. The CSA of the decay portion has a lot of information related to a structure's impulse response. However, for structural diagnostics, a single-number index is much more useful for a structural health monitoring system. In this section, we discuss the quantitative estimation of structural damage by using the CSA results of the decay portions. From the resonance decays of the CSA, we calculated the reverberant time $T_{R_{60}}$ of each resonance frequency for each condition. To calculate the reverberant time, significant resonance frequencies were selected from the CSA of condition 1 in Figure 7. The resonance frequencies of the other conditions were determined from the similar peaks of condition 1. Moreover, the reverberant time of each resonance frequency was calculated from the CSAs of the simulated observed signals in Figure 7. All reverberant times across conditions were normalized by the fundamental frequency's reverberant time of condition 1 . Using the reverberant times and resonance frequencies, the damping ratio $\zeta$ can be expressed as

$$
\zeta=\frac{6.9}{2 \pi f_{0} T_{R_{60}}},
$$

where $f_{0}$ is the resonance frequency and $T_{R_{60}}$ is the reverberant time of the resonance frequency $[9,10]$.

Figure 8 shows damping ratios estimated for each condition under a simulated observed signal. The upper panel shows the damping ratio across significant resonance frequencies. The damping ratio increases in going from condition 1 to condition 4 (as the structural degradation increases). This means that the average of the significant resonance frequency damping ratio could estimate structural degradation. The bottom panel shows a close-up of each resonance frequency. From these panels, it is clear that fine resonance information is difficult to estimate structural degradation because the resonance frequencies do not make simple transitions. In the second panel from the left, the resonance frequency rises and falls in going from condition 1 to condition 4 . The maximum damping ratio $\zeta_{\max }$ is also difficult to use as an indicator of structural degradation. $\zeta_{\max }$ is the fundamental frequency of the left panel, and the damping ratio of the fundamental frequencies have almost the same score because the fundamental frequency is robust to structural degradation. These results show that $\zeta_{\max }$ is not suited as an index 
of structural diagnostics. In contrast, the damping ratios of the other resonant frequencies show that the damping ratio increases as the structural degradation increases. As previously indicated, although the average damping ratio can be used to estimate structural diagnostics, the minimum damping ratio of each condition $\zeta_{\min }$ would also be suited to estimating structural degradation and is a simple way to diagnose structures.

\section{Summary}

We discussed how to estimate hidden resonance decays using CSAs under nonstationary noise conditions. The first experiment used two scale models to measure the impulse responses and to simulate degraded conditions. The CSAs of the impulse responses revealed clear resonant frequencies and decays. In addition, the peak of the fundamental frequency did not decay, even in very short frame lengths. This means that it is useful to estimate the decay portion of the observed signals because the observed signals do not necessarily have sufficiently long transient decay portions. The random burst noise analysis involved CSAs of different portions. The CSAs show that decay portions only reveal resonant decays. In contrast, the mean CSA of the decay portions shows clear resonant frequencies and their decays. Thus, the mean CSA is a better way to reveal these factors. Finally, we conducted a simulated observed signal experiment. The results showed that the resonance frequency and decay were very similar to the impulse response's CSAs. Moreover, the experiments accurately simulated the decay of the fundamental frequency. From these results, we can conclude that performing CSA on the decay portion is a good way to estimate the resonant frequencies and their decays in the presence of nonstationary noise without using spectral information about the external noise. We also discussed how to estimate structural damage by calculating the damping ratio from the CSA. We confirmed that the average damping ratio increases as the structural degradation increases. This indicates that structural changes can be monitored using a simple score derived from the CSAs. However, the CSAs have a lot of information on the structural conditions related to impulse responses. This means that CSAs might have many uses in structural diagnostics. Moreover, this method would be helpful for structural diagnostics using constantly monitored signals.

\section{Acknowledgments}

This work was supported by a Grant from the Japan Society for the Promotion of Science, KAKENHI (24760477). The authors would like to thank Yoshimutsu Hirata who helped in measuring the impulse responses with the scale model.

\section{References}

[1] P. J. Halliday and K. Grosh, "Maximum likelihood estimation of structural wave components from noisy data," Journal of the Acoustical Society of America, vol. 111, no. 4, pp. 1709-1717, 2002.

[2] J. G. McDaniel and W. S. Shepard, "Estimation of structural wave numbers from spatially sparse response measurements,"
Journal of the Acoustical Society of America, vol. 108, no. 4, pp. 1674-1682, 2000.

[3] Y. Hirata, "A method for monitoring invisible changes in a structure using its non-stationary vibration," Journal of Sound and Vibration, vol. 270, no. 4-5, pp. 1041-1044, 2004.

[4] Y. Hirata, M. Tohyama, M. Matsumoto, and S. Gotoh, "Health monitoring for a structure using its nonstationary vibration," Advances in Acoustics and Vibration, vol. 2010, Article ID 696512, 5 pages, 2010.

[5] Y. Takahashi, T. Taniguchi, and M. Tohyama, "Structural condition monitoring by cumulative harmonic analysis of random vibration," Advances in Acoustics and Vibration, vol. 2008, Article ID 261758, 8 pages, 2008.

[6] Y. Takahashi, S. Goto, and M. Tohyama, "Modal experiment on structural condition monitoring using cumulative harmonic analysis," Advances in Acoustics and Vibration, vol. 2012, Article ID 327135, 6 pages, 2012.

[7] J. M. Berman and L. R. Fincham, "The application of digital techniques to the measurement of loudspeakers," Journal of the Audio Engineering Society, vol. 25, no. 6, pp. 370-384, 1977.

[8] Y. Takahashi, M. Tohyama, and Y. Yamasaki, "Cumulative spectral analysis for transient decaying signals in a transmission system including a feedback loop," Journal of the Audio Engineering Society, vol. 54, no. 7-8, pp. 620-629, 2006.

[9] E. E. Ungar and E. M. Kerwin Jr., "Loss factors of viscoelastic systems in terms of energy concepts," Journal of the Acoustical Society of America, vol. 34, no. 7, pp. 954-957, 1962.

[10] R. F. Barron, Industrial Noise Control and Acoustics, Marcel Dekker, New York, NY, USA, 2003. 

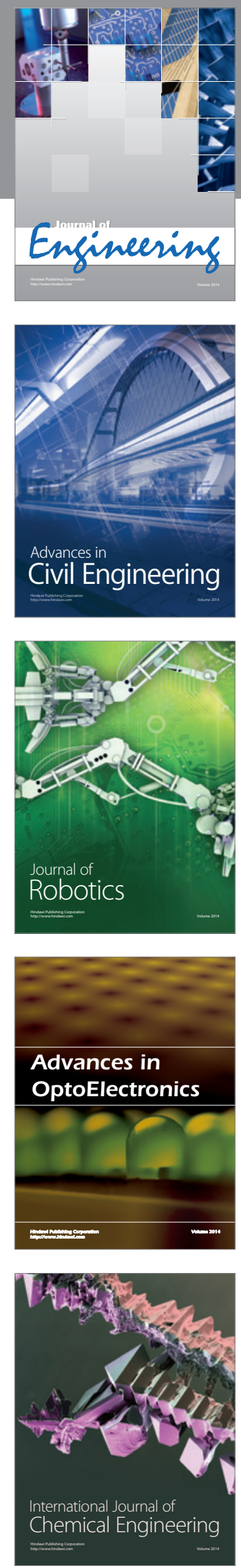

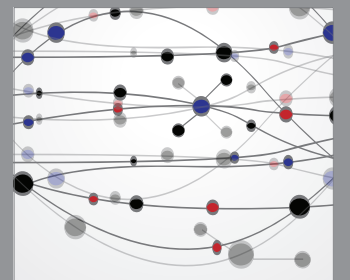

The Scientific World Journal
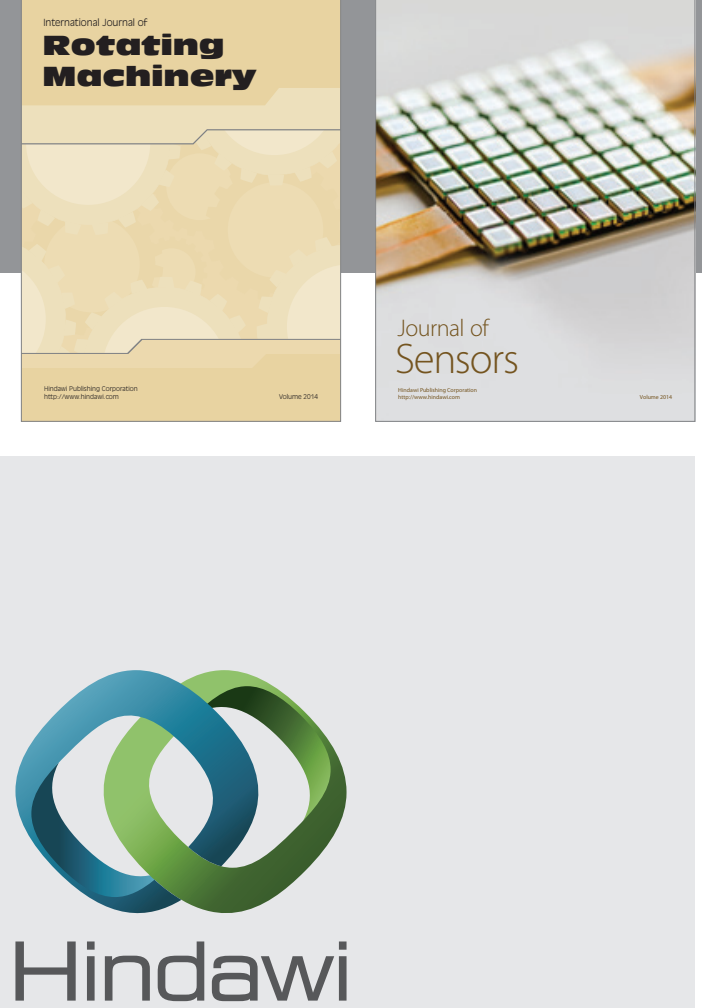

Submit your manuscripts at http://www.hindawi.com
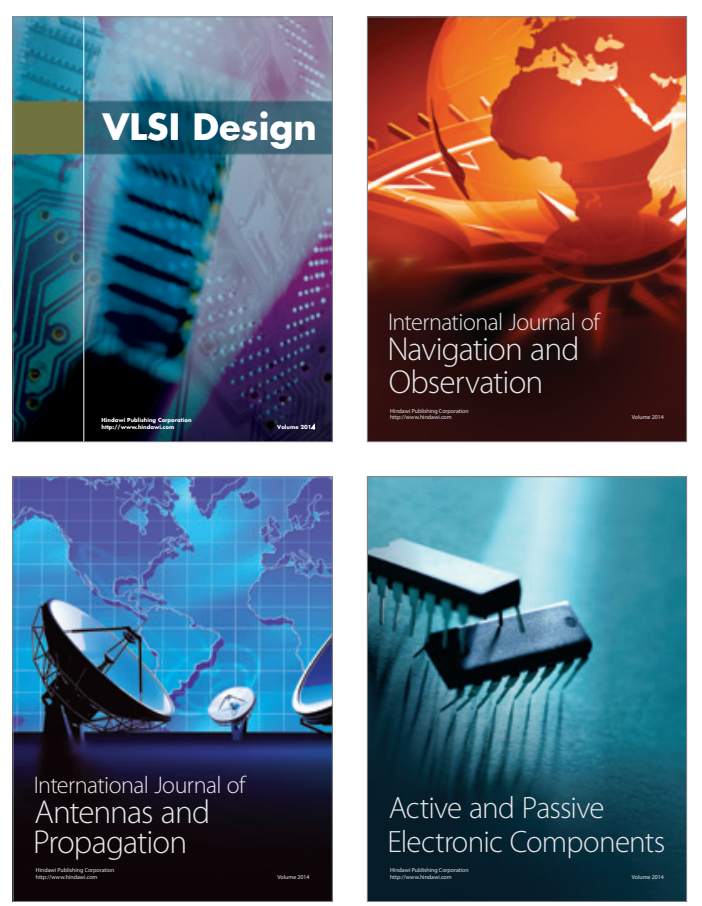
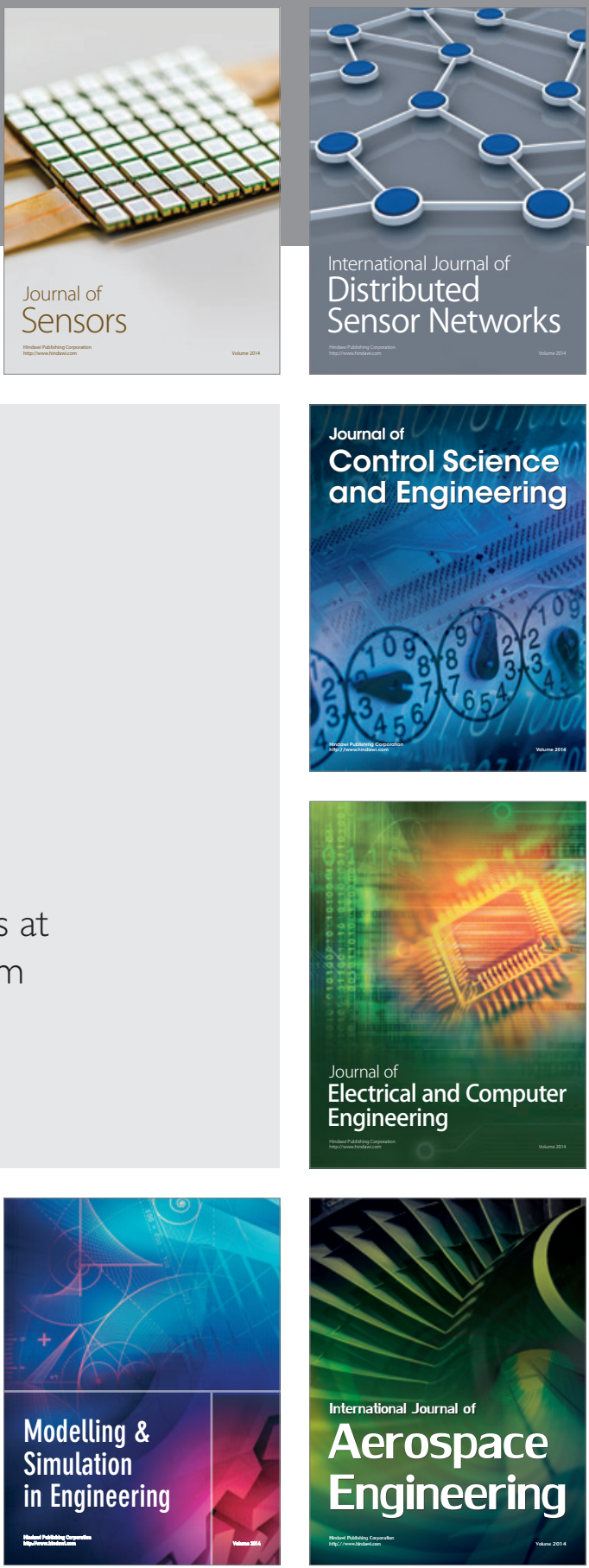

Journal of

Control Science

and Engineering
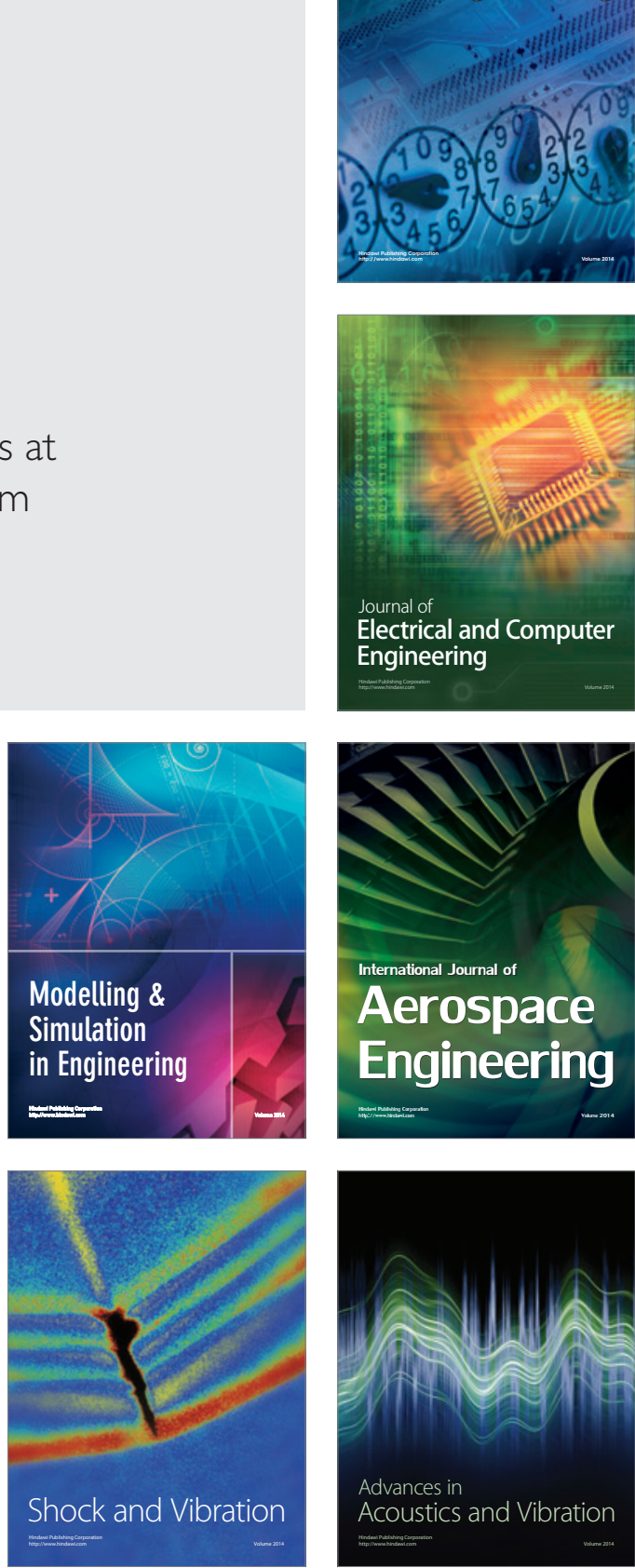